\title{
Distal pancreatic resection via laparo-endoscopic single site surgery - development of the technique
}

\author{
Maciej Michalik, Maciej Bobowicz, Paweł Lech, Michał Orłowski \\ General and Vascular Surgery Department, Ceynowa Hospital, Wejherowo, Poland
}

Videosurgery and other miniinvasive techniques 2010; 5 (4): 142-145 DOI: 10.5114/wiitm.2010.18827

\begin{abstract}
Aim: To present a novel technique of a laparo-endoscopic single site (LESS) distal pancreatic resection via the umbilicus with use of the QuadPort ${ }^{T M}$ Access System.

Material and methods: The technique of distal pancreatic resection through a $30 \mathrm{~mm}$ longitudinal incision in the umbilicus is described. Medial to lateral approach of pancreas preparation using a harmonic scalpel and regular laparoscopic instruments is applied. The described technique was successfully applied in a 26-year-old female patient with tumour of the pancreatic body and tail $(90 / 41 / 27 \mathrm{~mm})$. Pancreatectomy was followed by splenectomy due to infiltration of the splenic vessels.

Results: Total operative time was $160 \mathrm{~min}$. Blood loss was $800 \mathrm{ml}$. Initial wound size was $30 \mathrm{~mm}$. Splenic vein and artery infiltration forced augmentation of the wound to $80 \mathrm{~mm}$. The patient required only per request medication. Peristalsis returned spontaneously on the first postoperative day. Hospital stay was 4 days. There were no early perioperative complications. The patient required readmission due to electrolyte imbalance 7 weeks after the operation. Conclusions: The proposed operative technique of single incision laparoscopic distal pancreatectomy is difficult and challenging. Nonetheless, the authors proved that it is safe and feasible when performed by an experienced laparoscopic team.
\end{abstract}

Key words: distal pancreatic resection, single incision laparoscopic surgery, laparo-endoscopic single site surgery, LESS, minimally invasive surgery

\section{Introduction}

In the last two decades, minimally invasive surgery has been implemented in multiple surgical subspecialities. Pancreatic surgery remained on the sidelines of this process for a long time. Nevertheless, due to the unquestionable benefits of laparoscopic surgery, frequently it is seen as a method of choice in early tumours of the pancreatic body and tail [1]. Simultaneously, permanent progress in minimally invasive surgery led to development of the conception of laparo-endoscopic single site (LESS) surgery.
At present, LESS surgery is widely accepted in operations of the gallbladder, appendix and abdominal hernias [2]. Simultaneously, multiple reports have appeared on safe and successful LESS application in benign and malignant colorectal lesions, in obesity treatment and splenic resections [2, 3].

\section{Aim}

The aim of the study was to present a novel technique of minimally invasive laparoscopic distal pancreatic resection with single access surgery via one small umbilical incision. To the authors' knowledge 
this is the first report on LESS distal pancreatic resection via the QuadPort ${ }^{\mathrm{TM}}$ Access System.

\section{Methods}

\section{Preoperative period}

Standard preoperative antibiotic prophylaxis and antithrombotic prophylaxis as for laparoscopic pancreatectomy are administered.

\section{Patient's positioning}

The patient lies flat on the back in the anti-Trendelenburg position with legs separated. The operator stands between the legs of the patient. The assistant surgeon as well as the scrub nurse is situated on the left side of the patient.

\section{Operative procedure}

A $30 \mathrm{~mm}$ longitudinal incision via the umbilicus is made and open insertion of the QuadPort ${ }^{\mathrm{TM}}$, Olympus (Advanced Surgical Concepts, Wicklow, Ireland) is performed (Figure 1).

Pneumoperitoneum of $12 \mathrm{mmHg}$ is achieved. A $10-\mathrm{mm}$, straight $0^{\circ}$ laparoscopic camera is introduced and inspection of the abdominal cavity is done. One curved laparoscopic clinching grasping forceps (Olympus) is used to provide traction and avoid instruments clashing. Then using a laparoscopic grasper and 5-mm Harmonic Ace ${ }^{\circledR}$ Scalpel (Ethicon Endo-Surgery, Johnson \& Johnson) the greater omental sac is accessed. The pancreatic body and tail towards the splenic hilus are visualized. Preparation of the lower edge of the pancreatic body is performed starting from the pancreatic neck at the level of the mesenteric vein towards the pancreatic tail (medial to lateral approach) using a Harmonic Ace Scalpel, laparoscopic straight grasper and one laparoscopic curved grasper.

\section{Retrieval of the specimen}

The pneumoperitoneum is reduced and the QuadPort's booth is removed leaving the port's sleeve protecting the wound from possible port metastases during the pancreatic tumour removal.

\section{Drain and wound closure}

Haemostasis is assured and thorough inspection of the abdominal cavity is done. A Redon drain is inserted into the pancreatic bed and exenterated via a separate skin incision. Standard suturing material is used for wound closure.

\section{Splenectomy}

In the first case performed with the described technique, infiltration of the splenic vein and artery was discovered intraoperatively. The splenic artery was freed from the tumour tissue, but freeing of the splenic vein despite meticulous and lengthy efforts was unsuccessful. Therefore, a decision to perform splenectomy was made. The QuadPort was completely removed. The incision was augmented to $8 \mathrm{~cm}$ to facilitate exteriorization of resected tissues, especially the spleen.

The splenic artery and vein were clipped with size M Hem-o-lok ${ }^{\circledR}$ Ligation System (Teleflex Medical) and dissected with a harmonic scalpel. Splenic ligaments were freed and splenectomy completed.

Further operative steps followed the protocol presented above.

\section{Results}

The described technique was successfully applied in a 26-year-old female patient (BMI about $18 \mathrm{~kg} / \mathrm{m}^{2}$ ) with tumour of the pancreatic body and tail (90/41/27 mm). Despite having three CT-guided fine needle biopsies done, the aetiology of the tumour was unknown at the time of surgery. American Society of Anesthesiology (ASA) Score was II due to comorbid asthma with no previous abdominal surgery. Total operative time was $160 \mathrm{~min}$. The proximal pancreatic resection margin was $15 \mathrm{~mm}$. Blood loss was $800 \mathrm{ml}$. Initial wound size was $30 \mathrm{~mm}$. Splenic artery

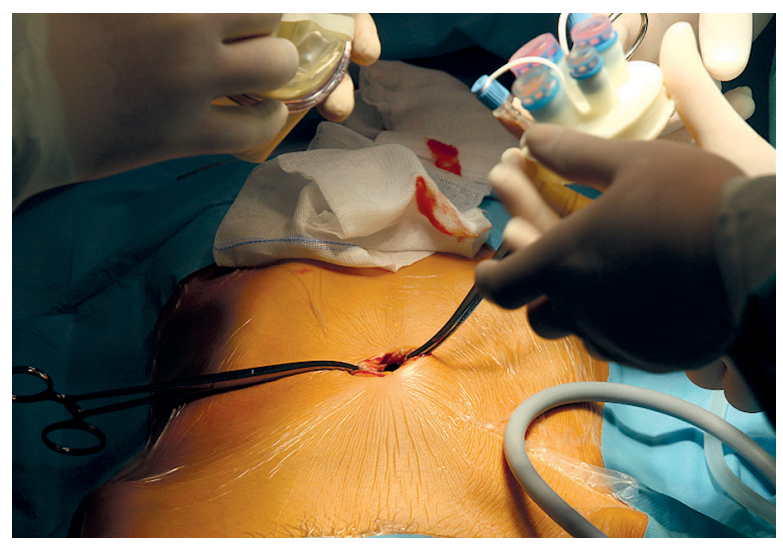

Figure 1. Open insertion of the QuadPort 


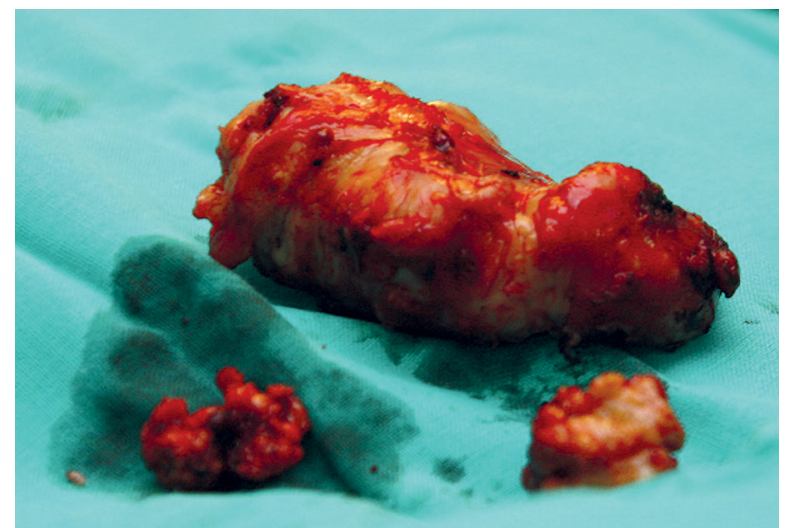

Figure 2. Resected body and tail of the pancreas

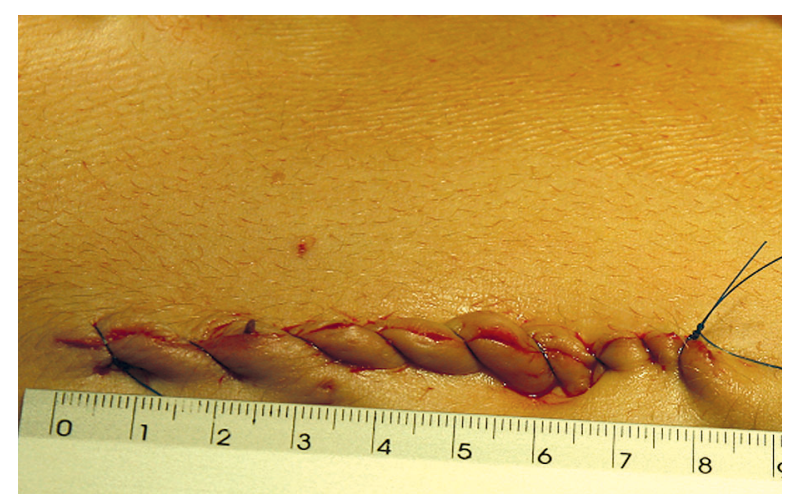

Figure 3. Final abdominal incision

and vein infiltration, need to perform splenectomy and need to exteriorize the spleen and indurated, cartilage-like pancreas (Figure 2) forced augmentation of the wound to $80 \mathrm{~mm}$ (Figure 3).

Postoperatively, the patient required only per request analgesics. Spontaneous return of peristalsis took place on the first postoperative day, when a liquid diet was started. Hospital stay was 4 days. Histopathological examination of the specimen revealed an inflammatory tumour. There were no early (30 days) perioperative complications. The patient required readmission due to electrolyte imbalance 7 weeks after the operation. In the 3-month follow-up period there were no further adverse events recorded.

\section{Discussion}

Until recently, pancreatic resections have remained the domain of open surgery. The scale of technical difficulties followed by the risk of complications required huge technical progress to be made before wide acceptance of minimally invasive laparoscopic techniques in pancreatic surgery. From the first report on laparoscopic pancreatic resection by Gagner et al. in 1996 [4] until 2005 there were only isolated case reports available. Since the multicentre retrospective analysis of 127 cases performed by Mabrut et al. [5] further reports on safety and feasibility of laparoscopic pancreatic resections involving larger groups of patients have started to appear. Kim et al. in their single institution retrospective analysis demonstrated the advantage of laparoscopic distal pancreatectomy compared to open procedure in terms of earlier resumption of diet and earlier discharge [6]. The authors also found no difference between open and laparoscopic approach with regard to the operative times, frequency of perioperative morbidity and mortality, and treatment costs. Further comparative studies and systematic reviews proved the laparoscopic approach to be superior also in terms of shorter operative times and decreased blood loss [7, 8].

Most of the studies on application of LESS have proved the feasibility and safety of this approach [913]. Nonetheless, due to the complexity of single access surgery there have been no successful cases of pancreatic resections in the available literature so far. The authors, before applying LESS in distal pancreatic resection (DPR), performed several laparoscopic DPRs as well as several LESS operations including cholecystectomies, right hemicolectomy [3] and total mesorectal excision as well as some transvaginal and transgastric NOTES operations [14] to achieve technical proficiency in minimal access surgery.

The presented technique requires a QuadPort Access System ${ }^{\circledR}$ that allows use of a $10-\mathrm{mm}$ laparoscopic camera and three operative instruments. The design of the port enables more freedom of movement than other commercially available single access ports. It also allows the use of regular, straight, laparoscopic instruments available in a laparoscopic surgery centre, therefore decreasing costs of the operation. There was only one curved laparoscopic grasper used to provide better triangulation and to avoid instruments' clashing - mainly outside the abdominal cavity (Figure 4). The port was located directly above the pancreatic field for better traction and visualization.

In the presented case, the initial umbilical incision was $30 \mathrm{~mm}$ long and would have allowed safe performance of the distal pancreatic resection with splenic preservation if not for the intraoperative diagnosis of splenic vein and artery infiltration. The 
splenic artery was freed from the tumour tissue but freeing of the splenic vein despite meticulous and lengthy efforts was unsuccessful. Therefore, the incision was augmented to $80 \mathrm{~mm}$ to facilitate safe splenectomy, oncological clearance (at this stage the tumour aetiology was uncertain) and exteriorization of resected tissues. Total operating time of $160 \mathrm{~min}$ was partially attributable to the efforts to preserve splenic vessels and was comparable to or shorter than laparoscopic distal pancreatectomies described in the literature $[6,15,16]$. On the other hand, there was greater blood loss than in many series due to the fact of splenic vessels' infiltration and technical difficulties of freeing them. Although, thanks to use of the harmonic scalpel, proximal pancreatic stump reinforcement with sutures was not required, the authors recommend Redon drain placement to avoid collection of any pancreatic secretions.

\section{Conclusions}

The proposed operative technique of laparoendoscopic single site distal pancreatectomy is difficult and challenging. Nonetheless, the authors proved that it is safe and feasible when performed by an experienced laparoscopic team. However, the patients should be carefully diagnosed and selected for the procedure to avoid intraoperative complications.

\section{References}

1. Merchant NB, Parikh AA, Kooby DA. Should all distal pancreatectomies be performed laparoscopically? Adv Surg 2009; 43: 283-300.

2. Romanelli JR, Earle DB. Single-port laparoscopic surgery: an overview. Surg Endosc 2009; 23: 1419-27.

3. Michalik M, Orlowski M, Frask A, et al. LESS (laparoendoscopic single-site surgery) right hemicolectomy. Videosurgery and other miniinvasive techniques 2009; 4: 164-7.

4. Gagner M, Pomp A, Herrera MF. Early experience with laparoscopic resections of islet cell tumors. Surgery 1996; 120: 1051-4.

5. Mabrut JY, Fernandez-Cruz L, Azagra JS, et al. Laparoscopic pancreatic resection: results of a multicenter European study of 127 patients. Surgery 2005; 137: 597-605.

6. Kim SC, Park KT, Hwang JW, et al. Comparative analysis of clinical outcomes for laparoscopic distal pancreatic resection and open distal pancreatic resection at a single institution. Surg Endosc 2008; 22: 2261-8.

7. Finan KR, Cannon EE, Kim EJ, et al. Laparoscopic and open distal pancreatectomy: a comparison of outcomes. Am Surg 2009; 75: 671-9.

8. Briggs CD, Mann CD, Irving GR, et al. Systematic review of minimally invasive pancreatic resection. J Gastrointest Surg 2009; 13 1129-37.

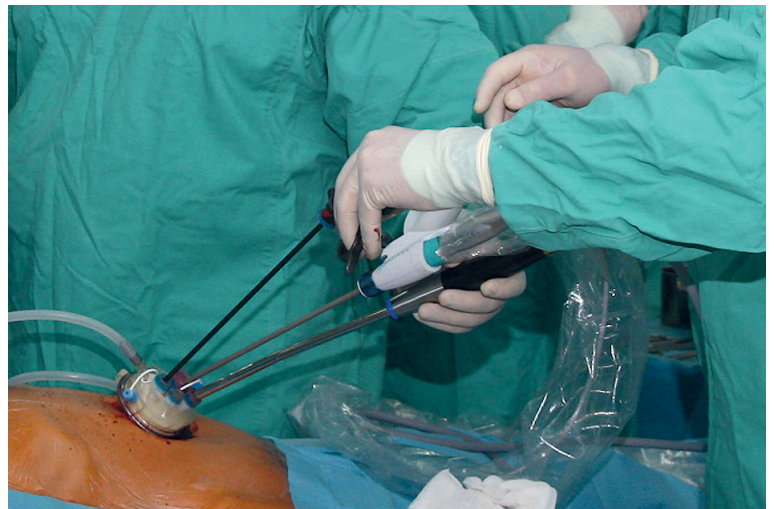

Figure 4. Parallel movement of the instruments

9. Losin M, Czauderna P, Golebiewski A, Stefanowicz J. Single incision laparoscopic adrenalectomy - initial experience. Videosurgery and other miniinvasive techniques 2010; 5: 104-6.

10. Budzynski A, Pedziwiatr M, Matlok M, et al. Preliminary experience with transperitoneal single incision laparoscopic surgery adrenalectomy. Videosurgery and other miniinvasive techniques 2010; 5: 87-92.

11. Cywinski J, Kuzdak K, Kolomecki K. One-incision approach (SILS) for retroperitoneal videoscopic adrenalectomy. Videosurgery and other miniinvasive techniques 2010; 5: 70-1.

12. Michalik M, Frask A, Trybull A, et al. Methods of treatment for gallbladder diseases. Videosurgery and other miniinvasive techniques 2009; 4: 121-5.

13. Kurpiewski W, Pesta W, Kowalczyk M, et al. SILS cholecystectomy - our first experiences. Videosurgery and other miniinvasive techniques 2010; 4: 91-4.

14. Michalik M, Orlowski M, Bobowicz M, et al. The First Report on Hybrid NOTES Adjustable Gastric Banding in Human. Obes Surg 2010 Mar 31. [Epub ahead of print].

15. Boutros C, Espat NJ, Somasundar P. Completely laparoscopic subtotal pancreatectomy with splenic artery preservation. J Gastrointest Surg 2010; 14: 171-4.

16. Nakamura Y, Uchida E, Nomura T, et al. Laparoscopic pancreatic resection: some benefits of evolving surgical techniques. J Hepatobiliary Pancreat Surg 2009; 16: 741-8. 